\title{
P02.82. Correction of the Omega-3 Index in women with metabolic syndrome by adding omega-3 supplements to a Mediterranean-style diet
}

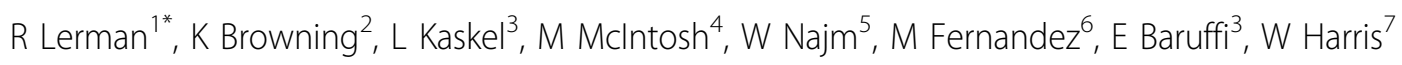

From International Research Congress on Integrative Medicine and Health 2012

Portland, Oregon, USA. 15-18 May 2012

\section{Purpose}

To determine the effects of a Mediterranean-style, lowglycemic-load (MLG) diet with and without omega-3 fatty acid supplementation on the Omega-3 Index (O3I) in women with metabolic syndrome.

\section{Methods}

One trial and one case series are reported here. The trial was a 12 -week multicenter study $(\mathrm{N}=56)$ testing the effects of MLG diet on the O3I, and the case series was conducted in the offices of two physicians and included 21 women who were given, in addition to a MLG diet recommendation, one of two omega-3 supplements for approximately 12 weeks. One supplement $(\mathrm{N}=12)$ provided 1980 mg EPA+DHA per day (High DHA, Metagenics Inc.), and the other supplement $(\mathrm{N}=9)$ provided $2880 \mathrm{mg}$ EPA+DHA daily (EPA-DHA 720, Metagenics Inc.). RBC fatty acid profiles were determined by gas chromatography and CVD risk factors by standard laboratory methods.

\section{Results}

In the clinical trial with the MLG diet alone, the O3I rose by $14.9 \%$ [from $4.35 \%$ to $5.00 \%(\mathrm{p}<0.0001)$ ], due largely to an increase in fish intake. RBC trans fatty acid, linoleic acid, and alpha-linolenic acid levels decreased. In the case series, in the 12 subjects who received High DHA, the O3I rose by $104.2 \%$ [from $3.6 \%$ to $7.3 \%(p<0.001)$ ] whereas in the 9 subjects who consumed EPA-DHA 720, the O3I rose by $99.8 \%$ [from $4.2 \%$ to $8.3 \%$ ( $<<0.001$ )]
The increase in the O3I per g of EPA+DHA given per day was $1.88 \%$ with High DHA and $1.44 \%$ with EPADHA 720.

\section{Conclusion}

The MLG diet led to a small improvement in the O3I but target levels were not achieved. Provision of 2-3 g/ day of EPA+DHA for 12 weeks increased the O3I by 3.7 - 4.1 percentage points and was sufficient to raise the O3I to cardioprotective levels. Therefore, an omega-3 supplement should be added to a MLG diet in patients with metabolic syndrome.

\section{Author details}

${ }^{1}$ Metagenics Inc., Gig Harbor, USA. ${ }^{2}$ Browning Family Medical Therapeutic Lifestyle Center, Riverside, USA. ${ }^{3}$ Deerpath Primary Care, Libertyville, USA. ${ }^{4}$ Dept. of Emergency Medicine, University of Florida, Jacksonville, USA. ${ }^{5}$ Dept. of Medicine, University of California, Irvine, Irvine, USA. ${ }^{6}$ Dept. of Nutritional Sciences, University of Connecticut, Storrs, USA. ${ }^{7}$ Sanford School of Medicine, University of South Dakota, Sioux Falls, USA.

Published: 12 June 2012

\section{doi:10.1186/1472-6882-12-S1-P138}

Cite this article as: Lerman et al:: P02.82. Correction of the Omega-3 Index in women with metabolic syndrome by adding omega-3 supplements to a Mediterranean-style diet. BMC Complementary and Alternative Medicine 2012 12(Suppl 1):P138.

${ }^{1}$ Metagenics Inc., Gig Harbor, USA

Full list of author information is available at the end of the article

(c) 2012 Lerman et al; licensee BioMed Central Ltd. This is an Open Access article distributed under the terms of the Creative Commons Attribution License (http://creativecommons.org/licenses/by/2.0), which permits unrestricted use, distribution, and reproduction in any medium, provided the original work is properly cited. 